\title{
Overexpression of COL24AI in Hepatocellular Carcinoma Predicts Poor Prognosis: A Study Based on Multiple Databases, Clinical Samples and Cell Lines
}

OncoTargets and Therapy

Long Yan

Feng Xu (D)

Chaoliu Dai

Department of General Surgery, Shengjing Hospital of China Medical University, Shenyang, Liaoning, People's Republic of China
Correspondence: Chaoliu Dai

Department of General Surgery,

Shengjing Hospital of China Medical

University, No. 36 Sanhao Street, Heping

District, Shenyang, Liaoning, People's

Republic of China

Tel + 861894025I697

Fax + 8624-96615

Email 384267542@qq.com
Purpose: Hepatocellular carcinoma (HCC) ranks as the second leading cause of cancerrelated deaths worldwide and its incidence tends to increase globally. Current biomarkers can hardly reflect diagnostic significance and therapeutic efficiency accurately. The role of collagen 24A1 (COL24A1) in HCC, as a member of the collagen family, remains unclear and needs to be proved.

Methods: HCC-associated RNA sequencing datasets were obtained from The Cancer Genome Atlas (TCGA), International Cancer Genome Consortium (ICGC) and Oncomine databases. $\mathrm{R}$ language was used to screen and analyze the differential expression genes (DEGs). Gene Ontology (GO) pathway analyses of DEGs were carried out using g:profiler. Then, the STRING database and cytoscape software were applied to estimate the correlations between DEGs. GEPIA and HCCDB database were used to verify and analyze these results. Finally, qPCR and immunohistochemistry staining on clinical samples and HCC cell lines were conducted to further verify the above results.

Results: The expression of COL24A1 in HCC tissues was significantly higher than that in normal hepatic $(\mathrm{NH})$ tissues. The analysis of TCGA and Oncomine database samples proved that COL24A1 expression was connected with tumor grade, tumor stage and pathologic stage. Meanwhile, the overall survival (OS) curve also validated that high expression of COL24A1 indicated poor prognosis. And the above results have been verified in our clinical samples and HCC cell lines. Furthermore, there is a significant increase of the expression of COL24A1 in various tumors. COL24A1, together with other interactive proteins, has a significant effect on the prognosis of HCC.

Conclusion: Overall, COL24A1 may be an oncogene in the development of HCC. Also, the overexpression of COL24A1 in HCC may help diagnose HCC and evaluate prognosis.

Keywords: COL24A1, hepatocellular carcinoma, database, differentially expressed gene, prognosis

\section{Introduction}

Hepatocellular carcinoma (HCC) is the second leading cause of cancer-related death worldwide, ${ }^{1}$ and its incidence is increasing globally. ${ }^{2}$ Without adequate diagnostic and prognostic biomarkers, the few effective treatments result in a poor prognosis of HCC, which is approximately $3-11 \%$ for 5-year survival rates. ${ }^{3}$ Owing to the limited understanding of the molecular mechanisms underlying HCC pathogenesis, developing specific and efficacious pharmacological strategies to treat, stop, or prevent 
progression to advanced stages is challenging. ${ }^{4}$ Therefore, this study aimed to find an effective diagnostic and therapeutic target, with the goal of prolonging the survival time of patients with HCC.

Collagens are a series of extracellular proteins that impart structural completeness to the connective tissue of almost every organ system. The collagen family includes 28 different $\alpha$-chain trimers or types that give rise to a large variety of suprastructures, such as fibrils, filaments, and networks. ${ }^{5,6}$ Collagen is also the main component of the tumor extracellular matrix, and it plays an important role in the development of tumors. ${ }^{7,8}$

Many studies have investigated the collagen family in HCC. Hepatocellular carcinoma is primarily associated with cirrhosis. Elastin and collagen proteins are abundant in liver cancer tissue, and their relationship with prognosis is gradually being emphasized. ${ }^{9}$ Dr Liu et al ${ }^{10}$ have indicated that activation of the PTK2-PI3K/Akt/mTOR pathway by COL4A1 and COL4A2 may contribute to hepatocarcinogenesis. Moreover, Dr Kimura et al ${ }^{11}$ have found that COL15A1 is up-regulated in HCC, and it contributes to the capillarization of HCC. Furthermore, Dr Zhang has demonstrated ${ }^{12}$ that collagen I enhances tumor cell proliferation and the epithelial-mesenchymal transition of $\mathrm{HCC}$, and is predictive of the progression of residual $\mathrm{HCC}$ cells after suboptimal heat treatment. Dr Ma et $\mathrm{al}^{13}$ have also found that COL1A1 promotes Slug-dependent epithelial-to-mesenchymal transition, thereby enhancing the invasion and metastasis of HCC.

COL24A1, which is predominantly expressed in bone tissue, is a poorly characterized member of the fibril-forming family of collagen molecules. ${ }^{14}$ This type of fibrocollagen may play an important role in regulating the physiological processes of bone and cartilage. The low level of COL24A1 expression observed in non-skeletal tissues (such as the brain, eyes, and other organs) suggests that this protein may play a broader role in organogenesis. ${ }^{15}$ In addition, Dr. Kiyoshi has demonstrated that the overexpression of the COL24A1 gene may be a valuable biomarker for prognosis in HNSCC. ${ }^{7}$

Certain markers, such as AFP, ${ }^{16}$ AFP-L3,${ }^{17}$ GCP3,${ }^{18}$ and MDK, ${ }^{19}$ have often been used in HCC diagnosis. Nevertheless, most of these markers are unlikely to exactly reflect the diagnostic and therapeutic efficiency. ${ }^{20}$ Thus, we evaluated the expression of COL24A1 in the TCGA, ICGC, and Oncomine databases and clinical samples to examine its clinical significance in HCC. In addition, we determined the expression of COL24A1 by using many analytical methods to further explore the molecular mechanisms of COL24A1 in the process of HCC.

\section{Materials and Methods TCGA Database Analysis}

To explore the expression of COL24A1 in HCC, we downloaded data for $374 \mathrm{HCC}$ and $50 \mathrm{NH}$ tissue samples in aggregate from the TCGA database (http://cancergenome.nih.gov/). Clinical variables, such as age, sex, pathologic stage, tumor grade, and AJCC TNM cancer stage, ${ }^{21,22}$ were used for the assessment of the correlation between the expression of COL24A1 and these parameters.

\section{ICGC and Oncomine Database Analysis}

A total of $240 \mathrm{HCC}$ and $202 \mathrm{NH}$ tissue samples were downloaded from the ICGC database (https://icgc.org), and the differential expression was examined. A total of 75 HCC tissue samples were collected from the Oncomine database (https://www.oncomine.org) and used to investigate clinical and prognostic differences.

\section{RNA Sequence Data Processing and Differential Expression Analysis}

DEseq, edgeR, and the limma package inR ${ }^{23-26}$ were used to confirm the differentially expressed mRNAs (DEmRNAs) between the $\mathrm{HCC}$ and $\mathrm{NH}$ tissues. To obtain sufficiently strong and convincing results in this large sample size, we used a false discovery rate (FDR) $<0.01$ and fold change $>10$ as thresholds indicating differential RNA expression. The heat map and volcano plots were constructed with the ggplot2 package in $\mathrm{R}^{27}$

\section{Differential Gene Function and Protein- Protein Interaction (PPI) Network Analysis} The Gene Ontology (GO) analysis of g:profiler (http://biit.cs. ut.ee/gprofiler/gost) was used to provide GO terms for DEmRNAs, and to identify significant biological functions $^{28}$ (FDR $\left.<0.01\right)$. To elucidate the molecular mechanisms of the cellular activities regulating tumorigenesis, the construction of a PPI network is imperative. The PPI network for screening genes was established with the STRING online database, and the interaction score was set to $\geq 0.4{ }^{29}$ In addition, the results were delineated in Cytoscape software.

\section{Validation of Differential Expression in GEPIA and HCCDB}

GEPIA was developed by Dr. Zhang's laboratory at Peking University. The data source of this tool is the TCGA 
database. We used this tool to analyze the expression of COL24A1 in different tumors and to explore the varying prognosis associated with differential expression of COL24A1 in all tumors.

HCCDB was provided by Tsinghua University to verify the repeatability of identification of HCC gene expression patterns and the annotation of transcripts. ${ }^{30}$ In this database, we analyzed the expression of COL24A1 and co-expressed genes in HCC.

\section{A}

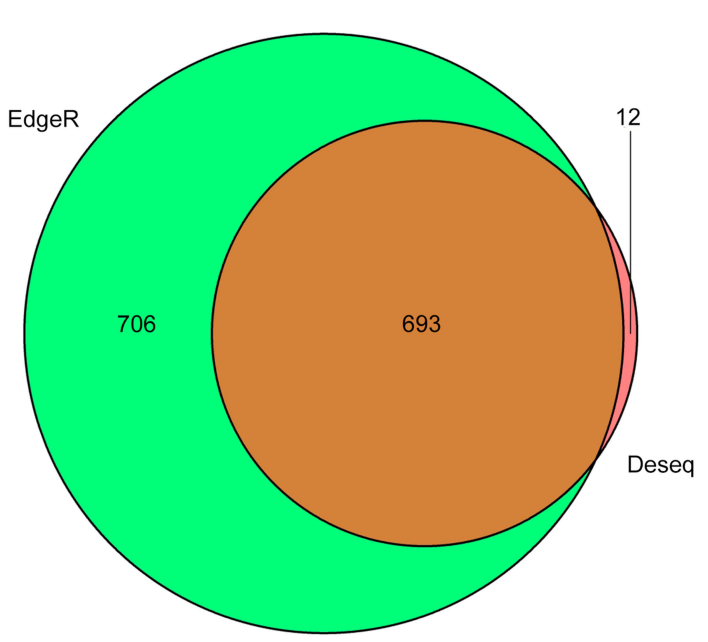

C

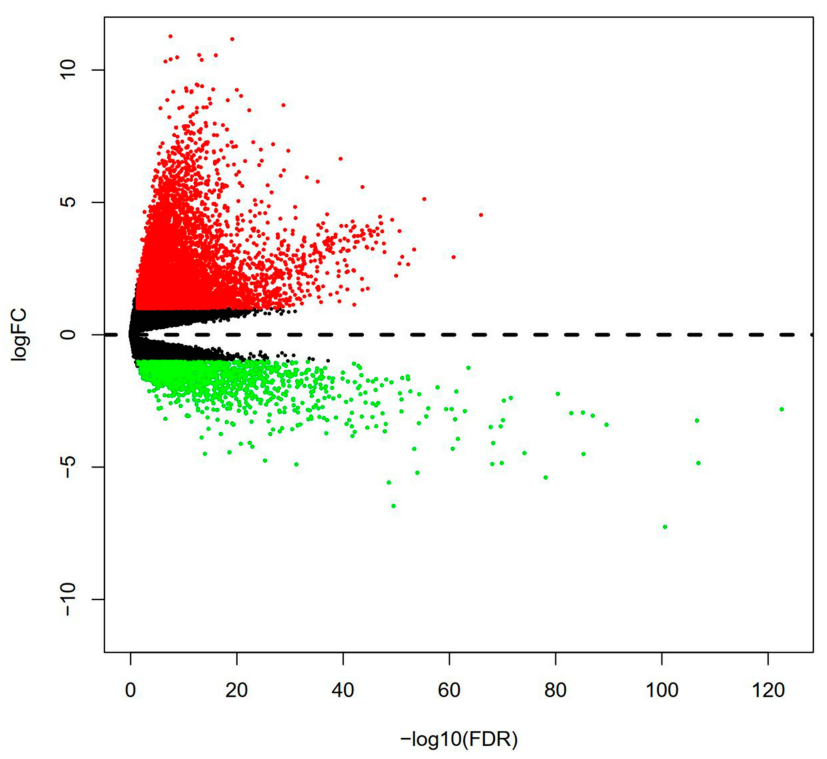

Expression of COL24AI in Clinical Samples and Cell Lines

To further identify the COL24A1 mRNA expression differences between $\mathrm{HCC}$ and $\mathrm{NH}$ tissues, we collected 80 pairs of clinical cases from the Shengjing Hospital of China Medical University between January 2017 and December 2018. All patients (61 males and 19 females; median age, 61.5; range, $37-77$ years old) were postoperatively diagnosed with HCC. We also cultured HepG2, Huh-7, and LO2 cells

\section{B}

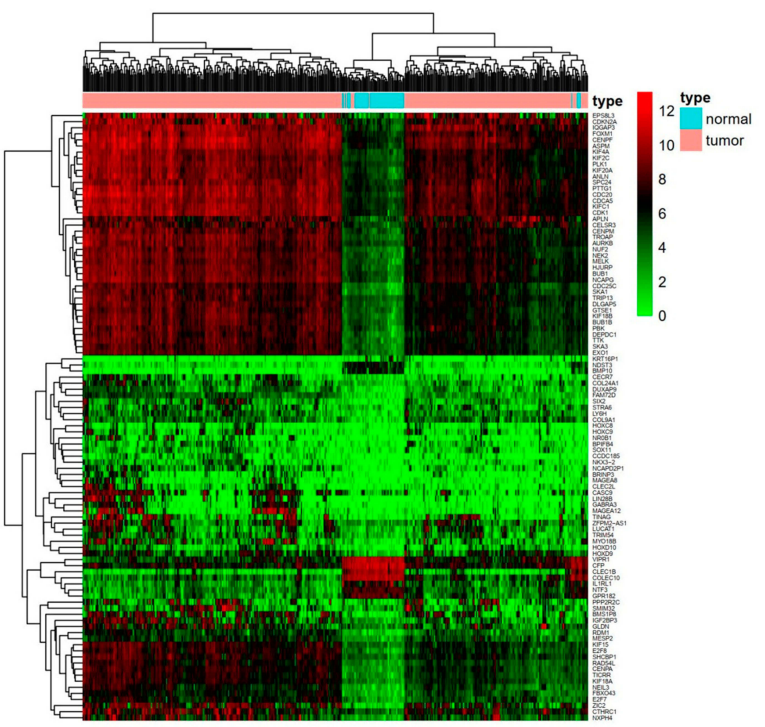

D

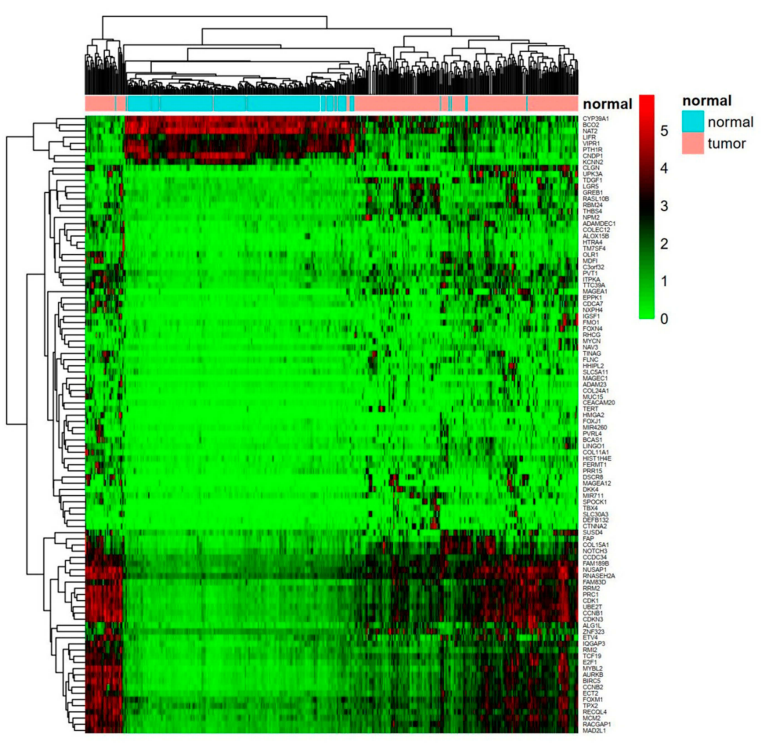

Figure I Differentially expressed genes in TCGA and ICGC. (A) A total 1399 differential genes were obtained by edgeR and 705 differential genes by DEseq (fold change $>10, P<0.01$ ). Venn diagram of data between the two statistical methods were 693 common differential genes. (B) We screened Heatmap of 100 signature genes in the differential genes of TCGA database. Each column represents a sample and each row represents one of the 100 genes. The expression levels of the 100 genes are shown in different colors, from green to red with increasing expression. (C) We detected 3621 differentially expressed genes (DEGs) in HCC from NH tissues by edgeR and screened volcano plot of DEGs. Each green dot shows a downregulated gene and each red dot shows an upregulated gene (fold change $>2$, P $<0.05$ ). (D) Heatmap of the I00 signature genes in the differential genes of ICGC database we screened. 
simultaneously. All the three cell lines were purchased from the Cell bank of Chinese Academy of Sciences, Shanghai. RNA from each sample, including cells, HCC, and NH tissues, was extracted according to the TaKaRa RNAiso Plus protocol. Next, the expression of COL24A1 was measured with TaKaRa TB Green ${ }^{\mathrm{TM}}$ Premix Ex $\mathrm{Taq}^{\mathrm{TM}}$ II (Takara Biomedical Technology, Beijing) with a Roche LightCycler ${ }^{\circledR}$ 480 system. In this case, $\beta$-Actin was used as an internal standard. The PCR cycle protocol used the following parameters: 1 cycle of $30 \mathrm{~s}$ at $95^{\circ} \mathrm{C}, 40$ cycles of $95^{\circ} \mathrm{C}$ for $5 \mathrm{~s}$, and $60^{\circ} \mathrm{C}$ for $20 \mathrm{~s}$. The fold change in expression was calculated with the $2^{-\Delta \Delta \mathrm{ct}}$ method. The primers used are as follows: COL24A1 forward: 5'-CCCAGCACGAATCTGCAAAG-3', reverse: 5'-GTCTGGCCACCAGCACTGAA-3'; and $\beta$-Actin forward: 5'-TGGCACCCAGCACAATGAA-3', reverse: 5'-C
Table I Clinical Association Between COL24AI Expression and Clinicopathological Variables in HCC Patients

\begin{tabular}{|l|l|l|l|}
\hline Variable & $\mathbf{9 5 \%} \mathbf{C l}$ & $\mathbf{T}$ & $\mathbf{P}$ \\
\hline Tumor Grade (G2-G4/GI) & $(\mathrm{I} 3.4996,55.7403)$ & 3.234 & $0.00 \mathrm{I}$ \\
TNM (II-IV/I) & $(8.4775,52.2537)$ & 2.038 & 0.043 \\
Stage T ( $\left.\mathrm{T}_{2}-\mathrm{T}_{4} / \mathrm{T}_{\mathrm{I}}\right)$ & $(\mathrm{I} .20 \mathrm{II}, 53.3549)$ & 2.065 & 0.040 \\
Pathologic Stage(II-IV/I) & $(8.4958,52.499 \mathrm{I})$ & 2.038 & 0.043 \\
\hline
\end{tabular}

TAAGTCATAGTCCGCCTAGAAGCA-3'. Ethical approval was provided by the Shengjing Hospital of China Medical University Ethics committee.

COL24A1 protein levels were measured by immunohistochemistry (IHC). We collected $40 \mathrm{HCC}$ clinical cases from the Shengjing Hospital of China Medical University from January 2015 to December 2016. All patients (32 males and
A

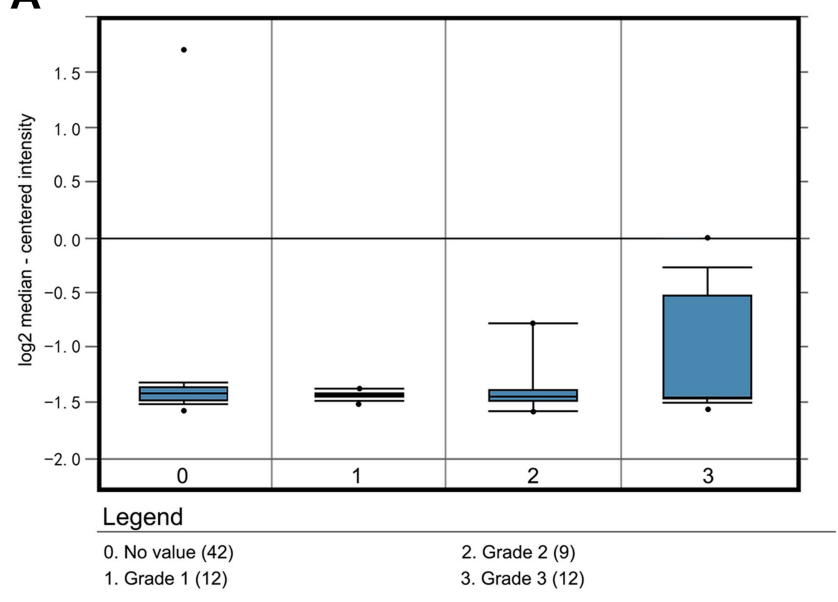

B

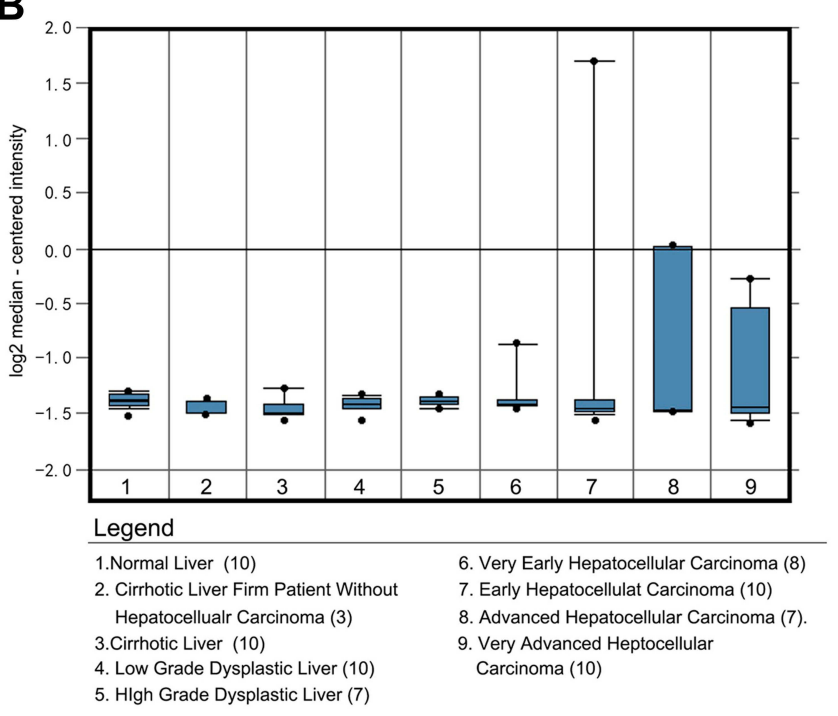

D

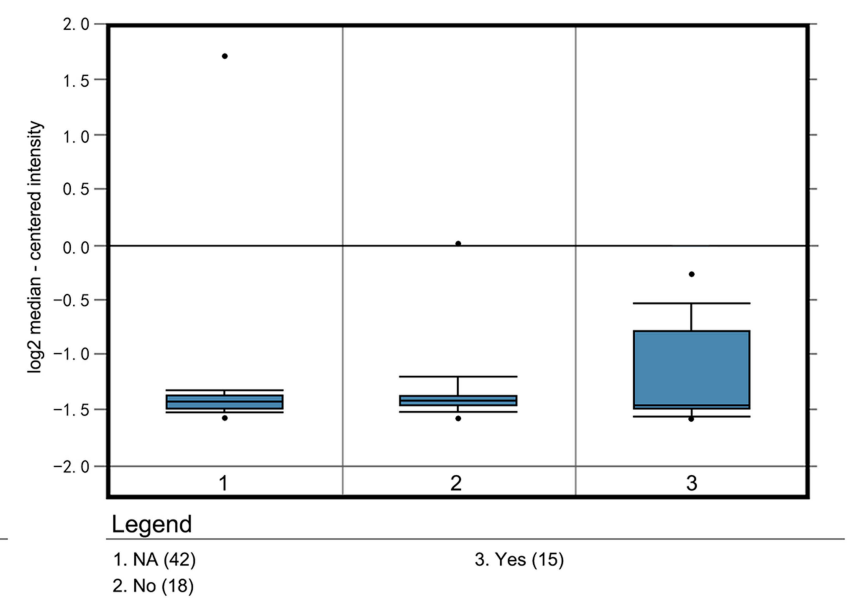

Figure 2 COL24AI expression analysis in 75 samples in Oncomine. (A) The COL24AI expression in different grade of HCC. (B) The COL24AI in different HCC stage. (C) The different COL24AI expression in HCC with vascular invasion. (D) The different COL24AI expression in HCC with or without satellites. 
8 females; median age, 60.5 years; range, $36-85$ years old) were diagnosed with HCC during postoperative examinations. All specimens were HCC tissues. The specimens were embedded in paraffin, and all the tissue blocks were cut into $4 \mu \mathrm{m}$ thick sections. The rabbit anti-COL24A1 antibody was provided by Biorbyt (California, United States. 1:2000). The expression of COL24A1 in tumor tissues was estimated according to the comprehensive score of COL24A1 staining intensity and positivity rate. Immunostaining was recorded according to the following criteria: 1) staining intensity scores of A: no staining $=0$, weak $=1$, medium $=2$, strong $=3$, and 2) positive staining ratio of COL24A1, evaluated as B: range $0(<5 \%)$, range $1(5-50 \%)$ and range $2(>50 \%)$. The total score $(\mathrm{A} * \mathrm{~B})$ of COL24A1 expression was divided into low expression $(0,1,2)$ and high expression $(3,4,6)$ groups. Finally, we calculated the correlation between COL24A1 expression in HCC, and patient prognosis and survival time.

\section{Statistical Analysis}

Statistical analysis, including receiver operating characteristic (ROC), Kaplan-Meier survival analyses, independent sample $T$-tests and $\chi^{2}$ tests were performed in SPSS 23.0. Univariate and multivariate analyses were based on Cox proportional hazard regression models. $\mathrm{P}<0.05$ was considered to indicate a statistically significant difference.

\section{Results \\ TCGA Filtering}

A total of 424 samples from the TCGA database for HCC were used. Two statistical methods in R, DEseq and edgeR, were used to screen differentially expressed genes (DEGs). Venn maps (Figure 1A), heat maps (Figure 1B), and volcano plots (Figure 1C) were constructed for these two groups of DEGs. We selected COL24A1 by combining the results of database screening and the relevant literature. With DEseq statistics, the expression fold change was 15.9 between HCC and $\mathrm{NH}$ tissues, and the FDR was 0.0003. Meanwhile, with edgeR statistics, the COL24A1 expression fold change was 15.52 between HCC and NH tissues, whereas the FDR was $3.7^{*} 10^{-15}$. The difference was clear and significant. Moreover, the expression level of COL24A1 was relatively high, in the top one-third among all detected genes.

\section{ICGC Validation}

We also collected 442 samples from the ICGC database to confirm the COL24A1 expression differences between HCC and $\mathrm{HN}$ tissues. We constructed a heatmap (Figure 1D) of 100 significant DEGs, and the expression fold change in COL24A1 was 9.5 between HCC and NH tissues. The FDR was $4.71 * 10^{-13}$. Therefore, the clear difference observed in the TCGA database was also confirmed in the ICGC database.
A

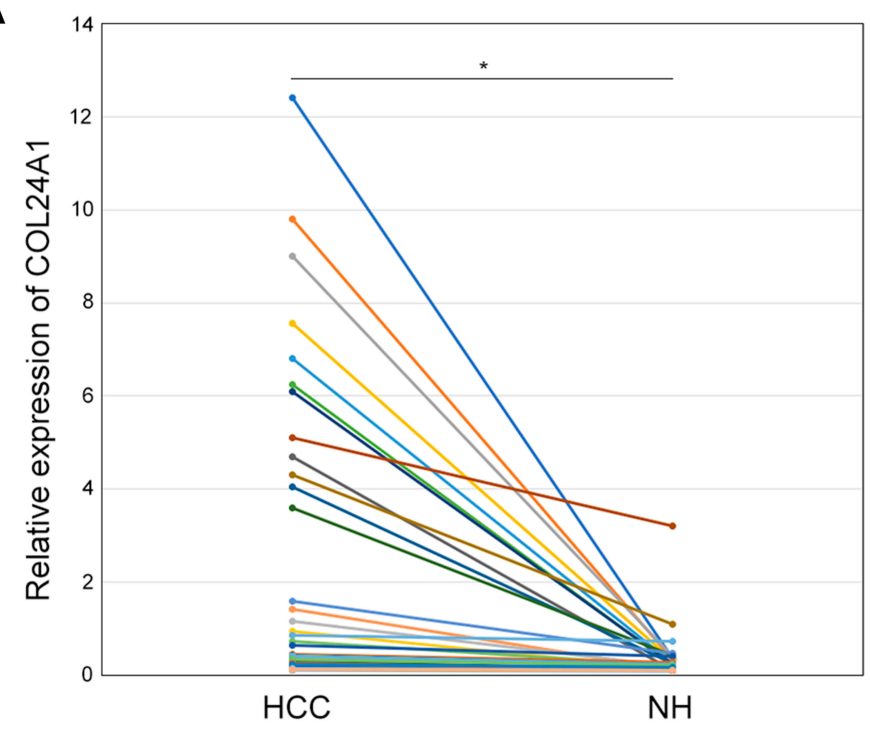

B

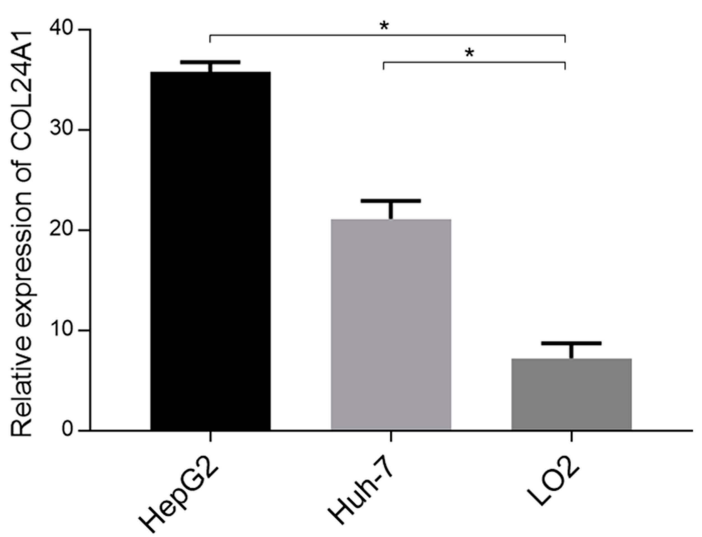

Figure 3 T-test of PCR results in 80 pair of clinical samples and 3 cell lines. (A) T-test of PCR results in 80 pair of clinical samples showed that the expression of COL24AI in $\mathrm{HCC}$ tissues was significantly higher than that in normal hepatic $(\mathrm{NH})$ tissues $(* \mathrm{P}<0.0 \mathrm{l})$. (B) The expression of COL24AI in HepG2 and Huh-7 was also significantly higher than that in LO2 cell line $(* \mathrm{P}<0.0 \mathrm{I})$ 


\section{COL24AI Expression}

The analysis of the independent sample $T$-tests of 424 TCGA samples revealed that COL24A1 expression was associated with tumor grade ( $\mathrm{T}=3.234, \mathrm{P}=0.001$; Table 1$)$, TNM stage $(\mathrm{T}=2.038, \mathrm{P}=0.043$; Table 1), $\mathrm{T}$ stage $(\mathrm{T}=2.065, \mathrm{P}=0.04$; Table 1) and pathologic stage $(\mathrm{T}=2.038, \mathrm{P}=0.043$; Table 1) on the basis of pathological diagnosis. We also collected statistical results for 75 tissue samples from the Oncomine database, including comparisons among different grades of HCC (Figure 2A), different stages of HCC (Figure 2B), cases with or without vascular invasion (Figure 2C), and cases with or without satellites (Figure 2D). As with our clinical specimens, the COL24A1 expression level was detected with PCR. The results indicated that COL24A1 was clearly overexpressed in HCC, rather than NH tissue (Figure 3A). In addition, in the three cell lines that we cultured, the expression of COL24A1 in the tumor cell lines HepG2 and Huh-7 was significantly higher than that in the normal hepatocyte cell line LO2 (Figure 3B). As shown in Figure 4, IHC was used to measure COL24A1 protein expression level, and the IHC staining of COL24A1 in HCC specimens is shown in Figure $4 \mathrm{~A}-\mathrm{D}$, revealing no, weak, moderate or strong

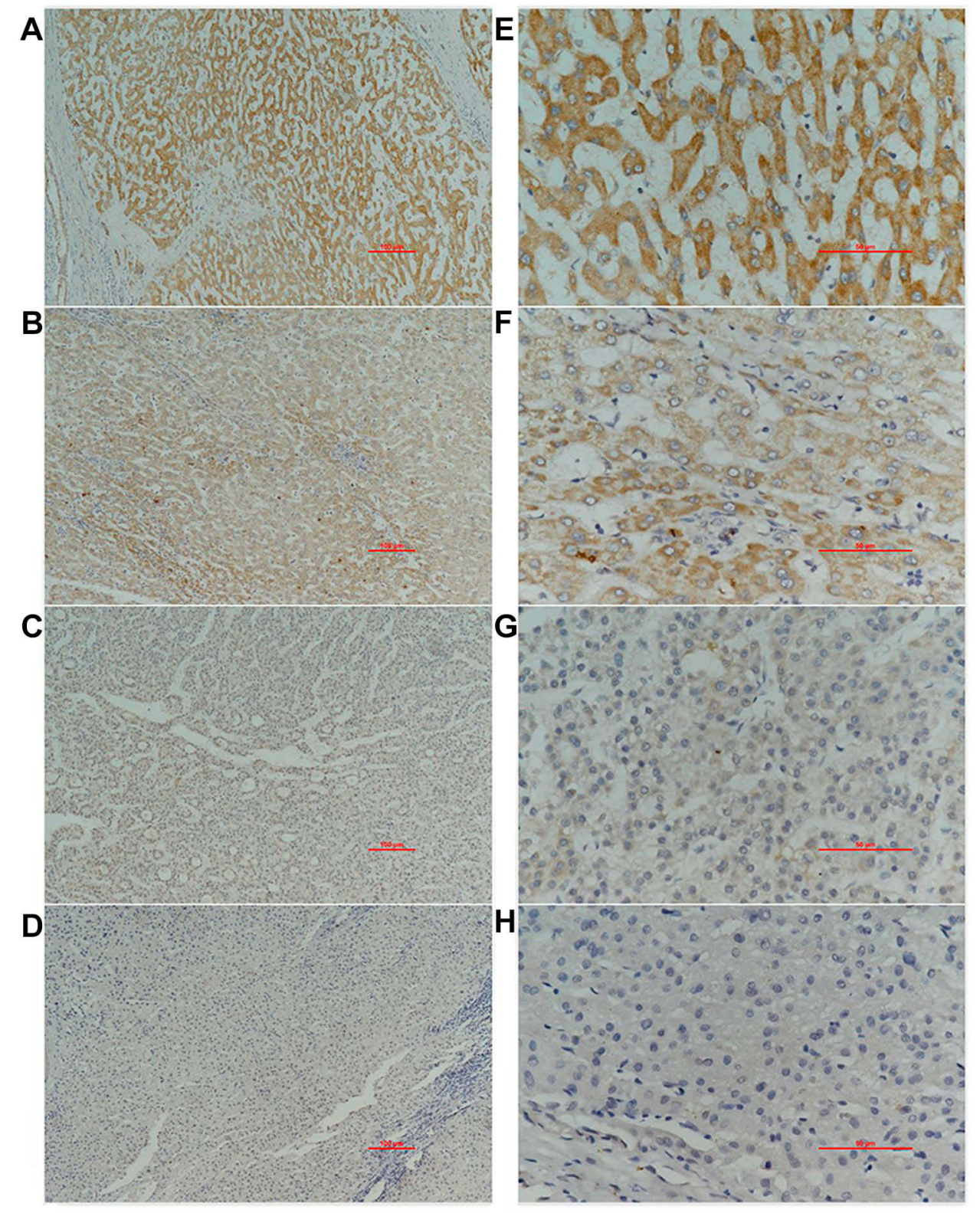

Figure 4 Immunohistochemical result in 40 clinical samples. Immunohistochemical staining of COL24AI in 40 HCC specimens: (A-D) represent no staining, weak, moderate, strong (XI00); (E-H) are high-power field images (X400). 
staining (X100); Figure 4E-H shows high-power field images (X400). Location analysis confirmed that COL24A1 was mainly concentrated in the cytoplasm in tumor cells. $\chi 2$ Tests were performed to assess the correlations between COL24A1 expression (low and high) and clinicopathological characteristics (Table 2). The results indicated that high expression of COL24A1 was predictive of later TNM stage and worse tumor grade, but was not significantly correlated with T stage and BCLC stage.

\section{Diagnostic and Survival Analysis}

Univariate Cox regression analysis indicated that the OS of HCC was markedly correlated with tumor grade, TNM stage, stage $\mathrm{T}$, pathologic stage, and COL24A1 expression (Table 3). However, multivariate analysis revealed that the expression of COL24A1 and the prognosis of HCC were also significantly (Table 4). Moreover, the OS of patients with HCC in both the TCGA (Figure 5A) and Oncomine (Figure 5C) databases, and 40 clinical cases collected from our hospital (Figure 5D), were analyzed with Kaplan-Meier plotter, and the results indicated that high COL24A1 expression was correlated with shorter OS in patients with HCC.

Table 2 Clinical Association Between COL24AI Expression and Clinicopathological Variables in HCC Clinical Samples

\begin{tabular}{|c|c|c|c|c|}
\hline \multirow[t]{2}{*}{ Variable } & \multirow[t]{2}{*}{ Number } & \multicolumn{2}{|c|}{ COL24AI Expression } & \multirow[t]{2}{*}{ P-value } \\
\hline & & Low & High & \\
\hline Age & & & & 0.616 \\
\hline$>60$ & 17 & 8 & 9 & \\
\hline$\leq 60$ & 23 & 9 & 14 & \\
\hline Gender & & & & 0.038 \\
\hline Male & 32 & 11 & 21 & \\
\hline Female & 8 & 6 & 2 & \\
\hline T Stage & & & & 0.356 \\
\hline TI & 30 & 14 & 16 & \\
\hline $\mathrm{T} 2-\mathrm{T} 4$ & 10 & 3 & 7 & \\
\hline TNM & & & & 0.03 \\
\hline I & 28 & 15 & 13 & \\
\hline II-IV & 12 & 2 & 10 & \\
\hline Tumor Stage & & & & 0.049 \\
\hline GI & 21 & 12 & 9 & \\
\hline G2-G4 & 19 & 5 & 14 & \\
\hline BCLC & & & & 0.072 \\
\hline A & 33 & 17 & 16 & \\
\hline B-D & 7 & I & 6 & \\
\hline
\end{tabular}

Table 3 Univariate Analysis of Prognostic Factors of HCC

\begin{tabular}{|c|c|c|c|}
\hline \multirow[t]{2}{*}{ Variable } & \multicolumn{3}{|l|}{ OS } \\
\hline & Hazard Ratio & $95 \% \mathrm{Cl}$ & P-value \\
\hline $\operatorname{Age}(\geq 60 /<60)$ & 0.887 & $(0.4358,1.805)$ & 0.741 \\
\hline Gender(Male/Female) & 0.5243 & $(0.2576,1.067)$ & 0.075 \\
\hline Pathologic Stage(II-IV/I) & 5.72 & $(2.186,14.97)$ & 0.000381 \\
\hline Stage $T(T 2-T 4 / T I)$ & 4.761 & $(1.944,11.66)$ & 0.000638 \\
\hline TNM (II-IV/I) & 5.517 & $(2.107,14.45)$ & 0.000507 \\
\hline Tumor Grade(G2-G4/GI) & 1.569 & $(0.2|26| I .58)$, & 0.659 \\
\hline COL24AI(High/Low) & 3.196 & $(1.47,6.949)$ & 0.00388 \\
\hline
\end{tabular}

Table 4 Multivariate Analysis of Prognostic Factors of HCC

\begin{tabular}{|l|l|l|l|}
\hline \multirow{2}{*}{ Variable } & \multicolumn{3}{|l|}{ OS } \\
\cline { 2 - 4 } & Hazard Ratio & $\mathbf{9 5 \%} \mathbf{~ C I}$ & P-value \\
\hline Pathologic Stage(II-IV/I) & $1.46 \mathrm{Ie}+07$ & $(0.000$, Inf $)$ & 0.9967 \\
Stage T(T2-T4/TI) & 0.746 & $(0.098,5.658)$ & 0.7769 \\
TNM (II-IV/I) & $4.124 \mathrm{e}-07$ & $(0.000$, Inf $)$ & 0.9971 \\
Tumor Grade(G2-G4/GI) & 0.6769 & $(0.084,5.423)$ & 0.659 \\
COL24AI(High/Low) & 0.896 & $(1.069,5.605)$ & 0.0341 \\
\hline
\end{tabular}

The disease-free survival analysis of COL24A1 in TCGA was also investigated (Figure 5B). The diagnostic value of COL24A1 in HCC was estimated with a ROC curve (X-axis: 1-specificity; Y-axis: sensitivity). An AUC value of 0.75 revealed a favorable diagnostic value $(\mathrm{P}<0.001$; Figure $6 \mathrm{~A})$.

\section{Bioinformatic Analysis}

According to GO analysis of the DEmRNAs in g:Profiler, COL24A1 was enriched in the terms cellular component and collagen trimer, which contains MARCO, FCN3, FCN2, COLEC10, COL6A6, CCBE1, COL15A1, COL25A1, COL9A1, CTHRC1, DCN, COL2A1, C1QL1, COL24A1, GLDN, and C1QL4. The adjusted P value was $3.796^{*} 10^{-4}$ (Figure 6B).

We input all the DEGs screened by Deseq into STRING and obtained 882 DEGs for PPI analysis in Cytoscape. The network was constructed with combined scores $\geq 0.4$ and contained 774 nodes and 8473 edges. Then, a functional module was identified with the MCODE plugin, which comprised seven nodes and 17 edges, including COL24A1, COL15A1, COL25A1, COL2A1, COL6A6, COL9A1, and DCN (Figure 6C). We also analyzed the prognostic value of the other six proteins according to the TCGA database, and we found that four of them are simultaneously closely associated with prognosis (Figure 7). The results also reveal the value of COL24A1 in protein interaction and prognosis. 
A

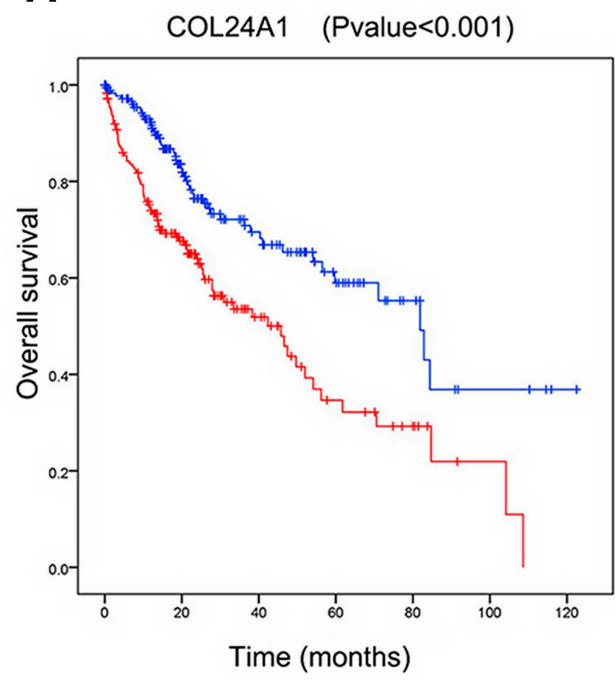

C

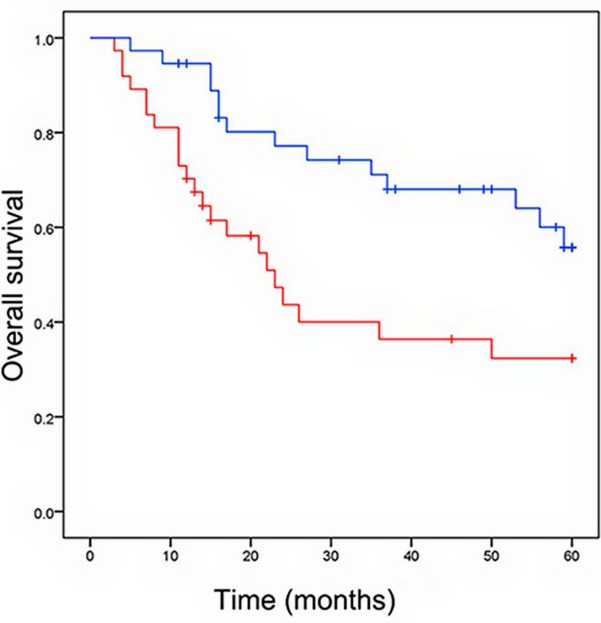

B

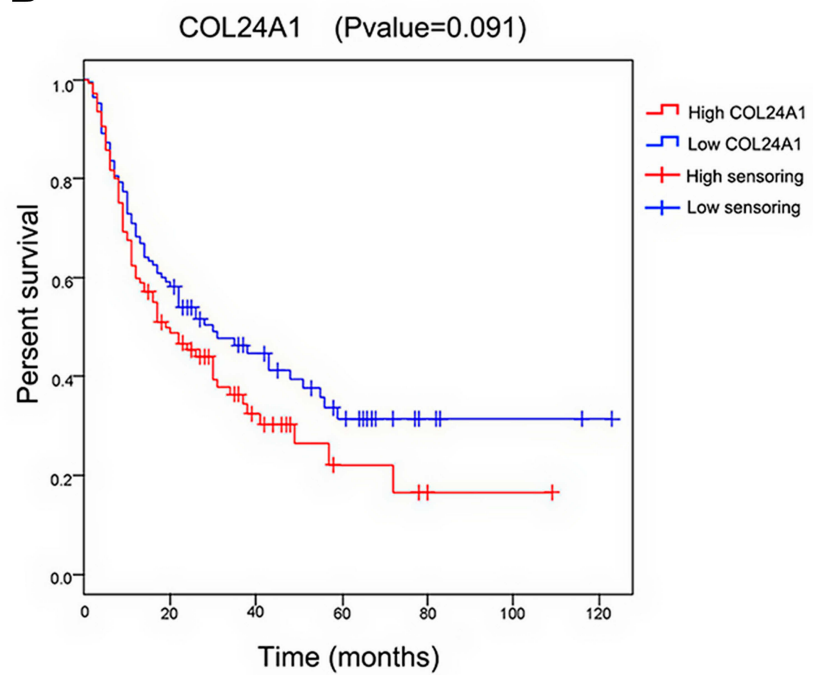

D

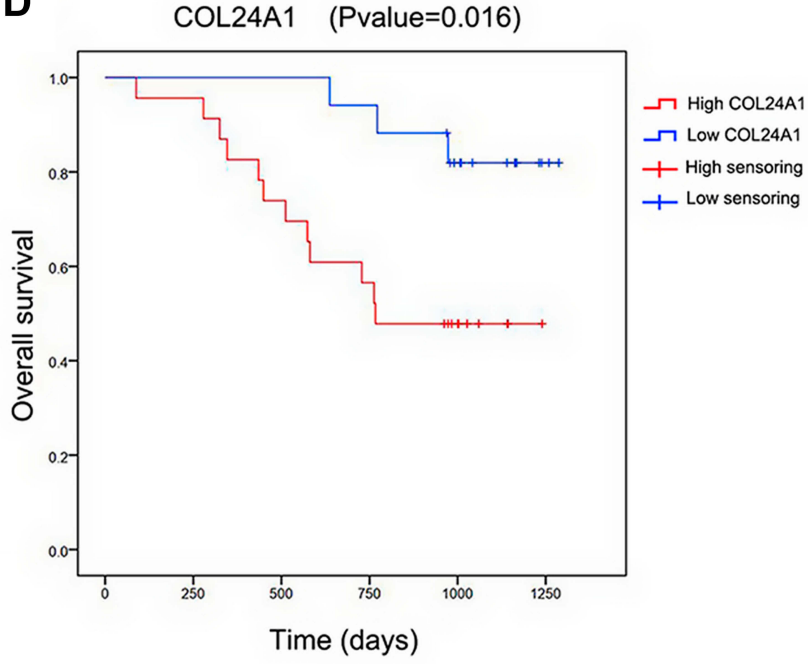

Figure 5 Survival analysis of COL24AI in databases and clinical cases. (A) Survival analysis of COL24AI in 374 samples in TCGA database demonstrated that high COL24AI expression was significantly associated with a reduced OS using Kaplan-Meier Plotter. (B) Disease free survival analysis of COL24AI in 374 samples in TCGA database did not demonstrate that high COL24AI expression was significantly associated with the reduced OS. (C) Survival analysis of COL24AI in 75 samples in Oncomine database demonstrated that high COL24AI expression was significantly associated with a reduced OS. (D) Survival analysis of COL24AI in 40 clinical cases demonstrated that high COL24AI expression was significantly associated with a reduced OS.

The modification of COL24A1 in 1424 cases of HCC in cBioPortal was investigated ${ }^{31,32}$ (Figure 6D). The results revealed that $2.1 \%(30 / 1424)$ of HCC cases presented COL24A1 modification, containing missense mutations (21/1424), amplifications (2/1424), truncating mutations (5/1424), and extensive deletions (2/1424).

Finally, we validated the differential expression of COL24A1 with the GEPIA tool and HCCDB database. In GEPIA, we found that the expression of COL24A1 in 10 tumor tissues - hepatocellular carcinoma, breast invasive carcinoma, cholangiocarcinoma, colon adenocarcinoma, head and neck squamous cell carcinoma, lung adenocarcinoma, lung squamous cell carcinoma, prostate adenocarcinoma, sarcoma, and pheochromocytoma/paraganglioma - was clearly higher than that in normal tissues (Figure 8A). In addition, we performed statistical analysis of the expression of COL24A1 in all tumors, and the OS curve again validated that high expression of COL24A1 was indicative of poor prognosis (Figure $8 \mathrm{~B}$ ). Then, in HCCDB, we demonstrated that the COL24A1 expression in $\mathrm{HCC}$ tissues was more significant than that in $\mathrm{NH}$ tissues in 8 of 10 clinical cohorts (Figure 9A). COL24A1 was expressed in various tissues, but its expression was low in normal liver tissues (Figure 9B) and significantly 
A

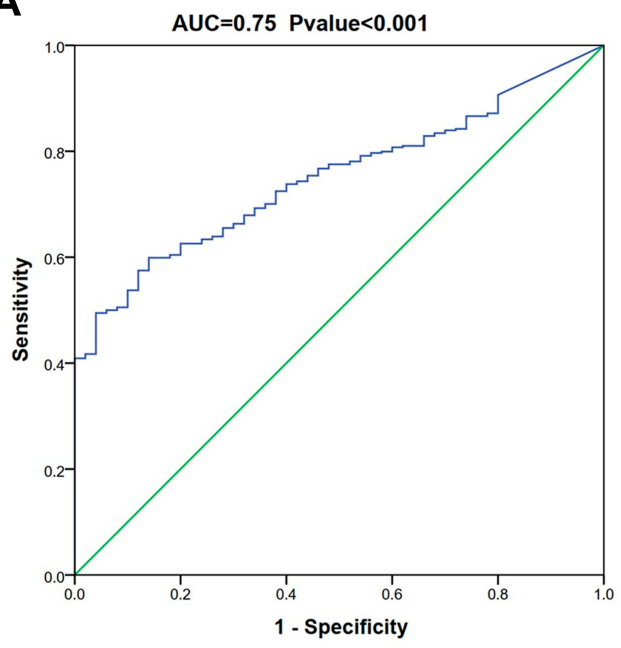

C

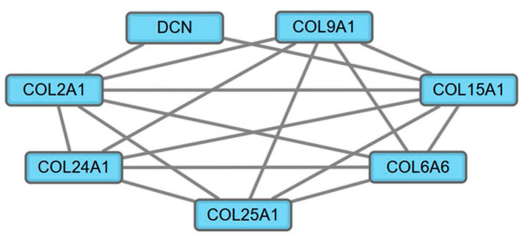

D

coL24A1 $2.1 \%$ | IIIHHHHHHHHHHHHHHH|
B

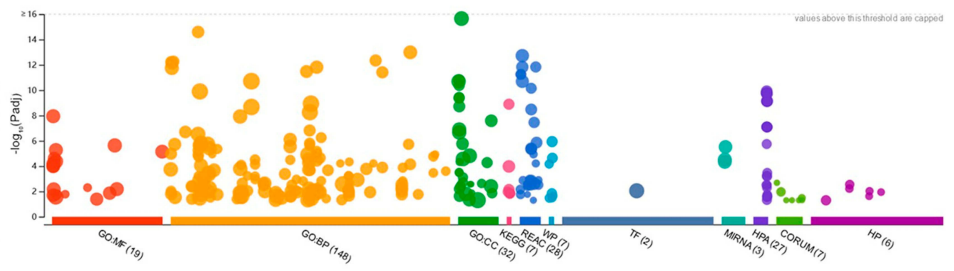

\begin{tabular}{l} 
Term name \\
\hline chromosome, centromeric region \\
condensed chromosome, centron
\end{tabular}

condensed chromosome, centromeric region

kinetochore

condensed chromosome kinetochore

condensed chromosome

chromosomal region

spindle

chromosome

condensed chromosome outer kinetochore

mitotic spindle

spindle microtubule

chromosomal part

condensed nuclear chromosome, centromeric region

spindle midzone

clear chromosome kinetochore

collagen trimer

kinesin complex

Ndc80 complex

condensed nuclear chromosome outer kinetoch

microtubule cytoskeleton

spindle pole

microtubule

chromosome passenger complex

midbody

microtubule associated complex

condensed nuclear chromosome

centralspindlin complex

GABA-A receptor complex

GABA receptor complex

intercellular bridge

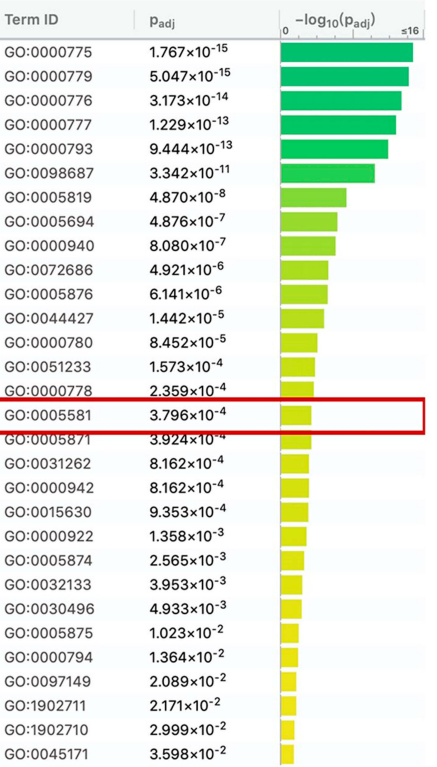

Figure 6 The ROC curve, GO analysis, PPI network analysis, alteration analysis of COL24AI. (A) The ROC curve of COL24AI expression in HCC, the curve indicated that COL24AI possessed a moderate diagnostic ability for GC (AUC $=0.75, P<0.001$ ). (B) GO analysis of the differentially expressed mRNAs in $\mathrm{g}$ :Profiler. (C) PPI network analysis of the differentially expressed mRNAs in STRING database. (D) A total of $2.1 \%$ of HCC cases exhibited COL24AI transformation in cBioPortal.

higher in HCC (Figure 9C). The HCC meta co-expression network is also summarized in this database (Figure 9D).

\section{Discussion}

HCC is a common malignant tumor worldwide, and it is often diagnosed in advanced stages, when the fatality rate is very high. ${ }^{33}$ At present, the diagnosis of hepatocellular carcinoma primarily depends on CT, MRI and other imaging methods, ${ }^{34}$ or some serological indicators such as AFP and AFP-L3. ${ }^{35}$ The overall detection rate remains relatively low. Therefore, determining effective diagnostic and prognostic targets for $\mathrm{HCC}$ is necessary. However, no systematic study on the diagnostic and prognostic value of COL24A1 in HCC has been reported to date.

As shown in two heat maps, the COL24A1 expression in $\mathrm{HCC}$ tissues in the two databases was significantly higher than that in NH tissues. In addition, as shown in Table 1, the association between COL24A1 expression and clinicopathological features was evaluated with independent sample
$T$-tests. The high expression of COL24A1 indicated more serious tumor invasion, poorer pathologic stage and later clinical stage, and these results were confirmed in our IHC analysis of 40 clinical samples, as shown in Table 2. Simultaneously, as illustrated in Figure 2, high expression of COL24A1 was also predictive of vascular invasion and the presence of satellites. The analysis of the TCGA and ICGC database suggested that COL24A1 has significantly higher expression in $\mathrm{HCC}$ than in $\mathrm{NH}$ tissues. The results of our analysis of 80 pairs of clinical samples with PCR tests were essentially the same. Therefore, COL24A1 was inferred to be an oncogene involved in the occurrence and development of HCC. Then, we chose two classic liver cancer cell lines, HepG2 and Huh-7, and one normal hepatocyte cell line, LO2, to further validate the results. As expected, the expression of COL24A1 in HepG2 and Huh7 was higher than that in LO2. We then used GEPIA to explore the expression of COL24A1 in different tumors, and we found that COL24A1 was highly expressed in at least 10 

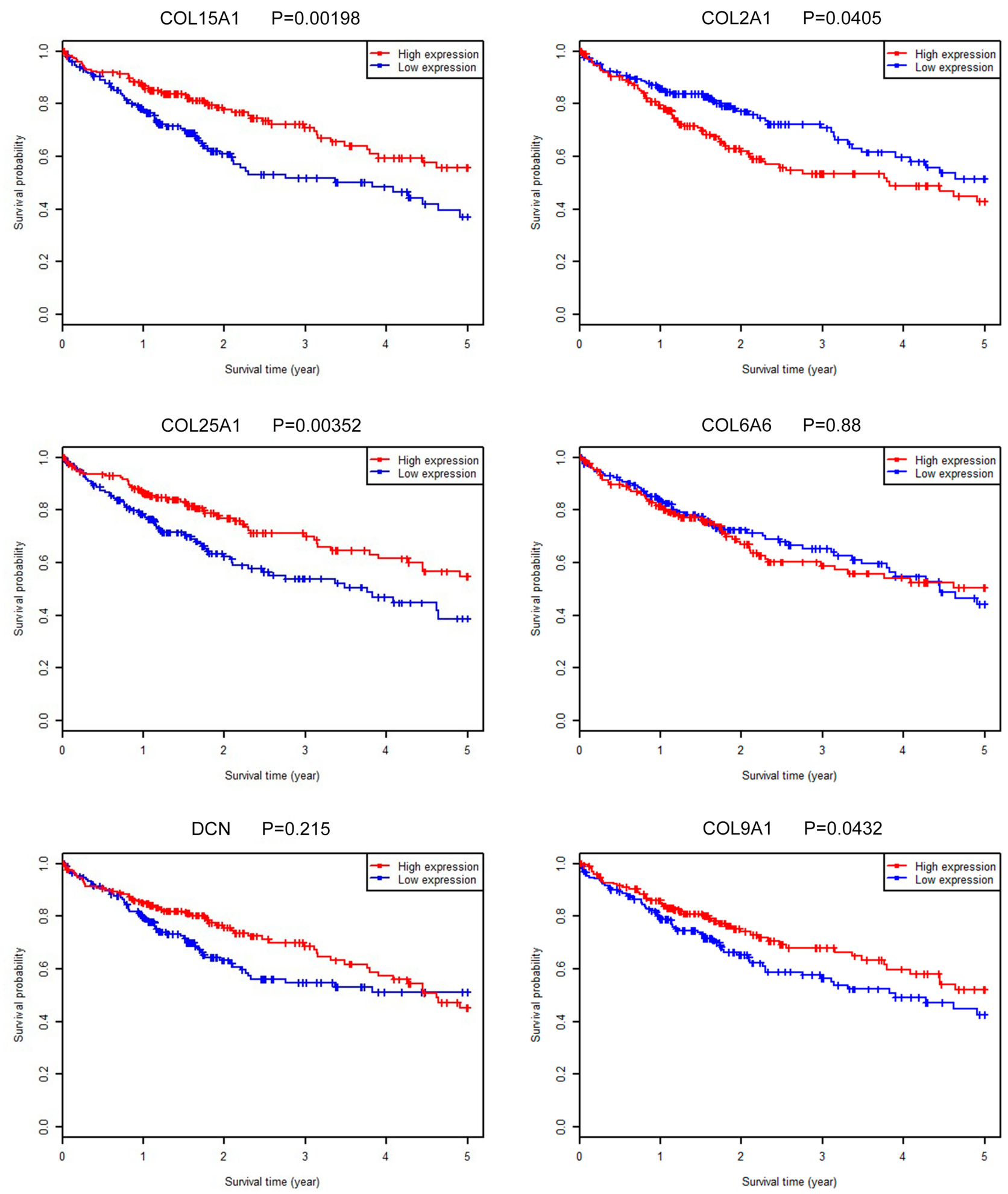

Figure 7 Survival analysis of COL5AI, COL25AI, DCN, COL2AI, COL6A6 and COL9AI in TCGA database. Survival analysis of COL5AI, COL25AI, COL2AI and COL9AI in TCGA database demonstrated that high expression of them were significantly associated with the reduced OS.

tumors; thus, also suggesting that COL24A1 expression might be common among different tumors in different organs. Finally, we grouped all tumors according to the expression of COL24A1 and analyzed the prognosis. The OS curve validated that high expression of COL24A1 indeed indicated a poor prognosis. This finding again 
A
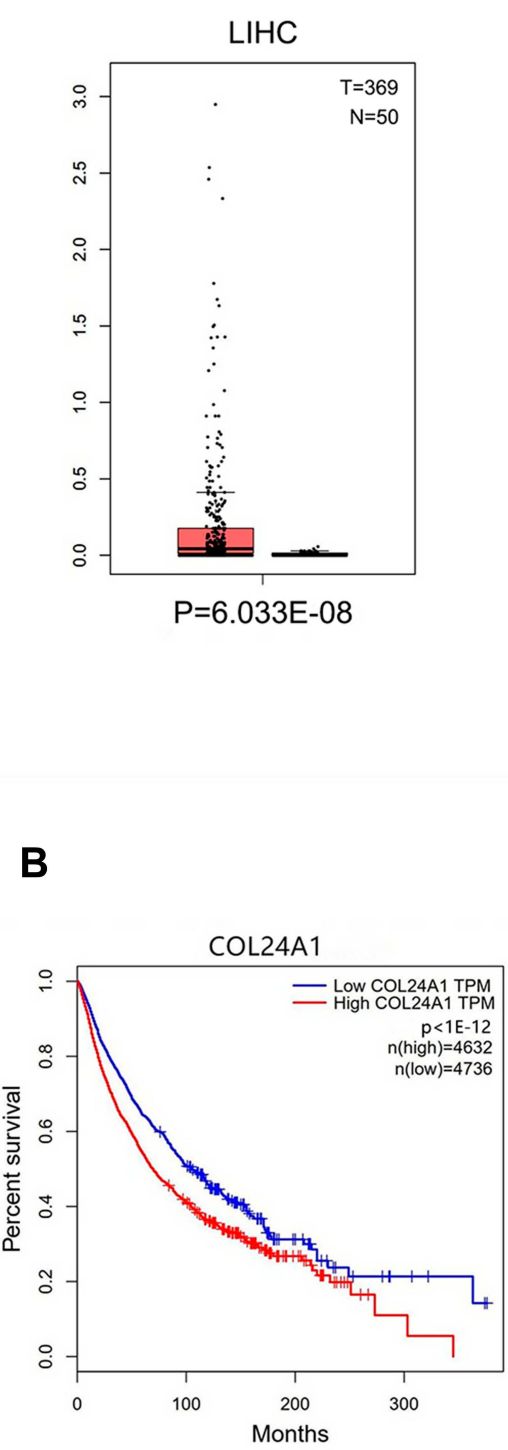

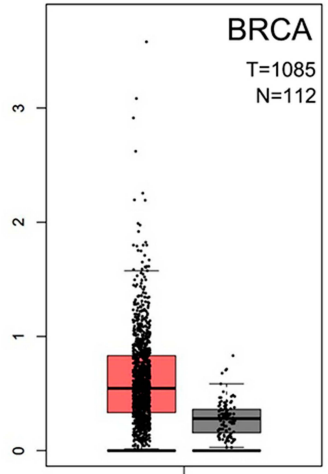

$\mathrm{P}<1 \mathrm{E}-12$

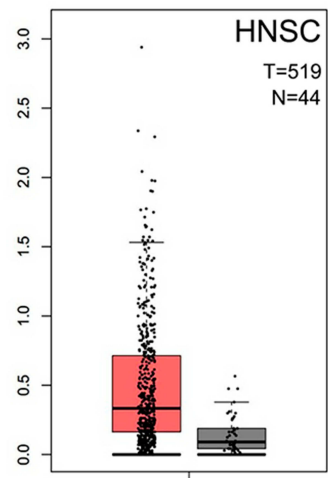

$\mathrm{P}<1 \mathrm{E}-12$

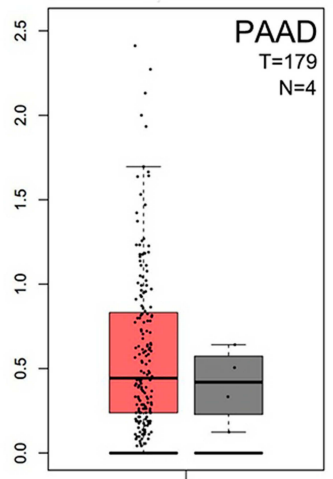

$\mathrm{P}=8.644 \mathrm{E}-03$
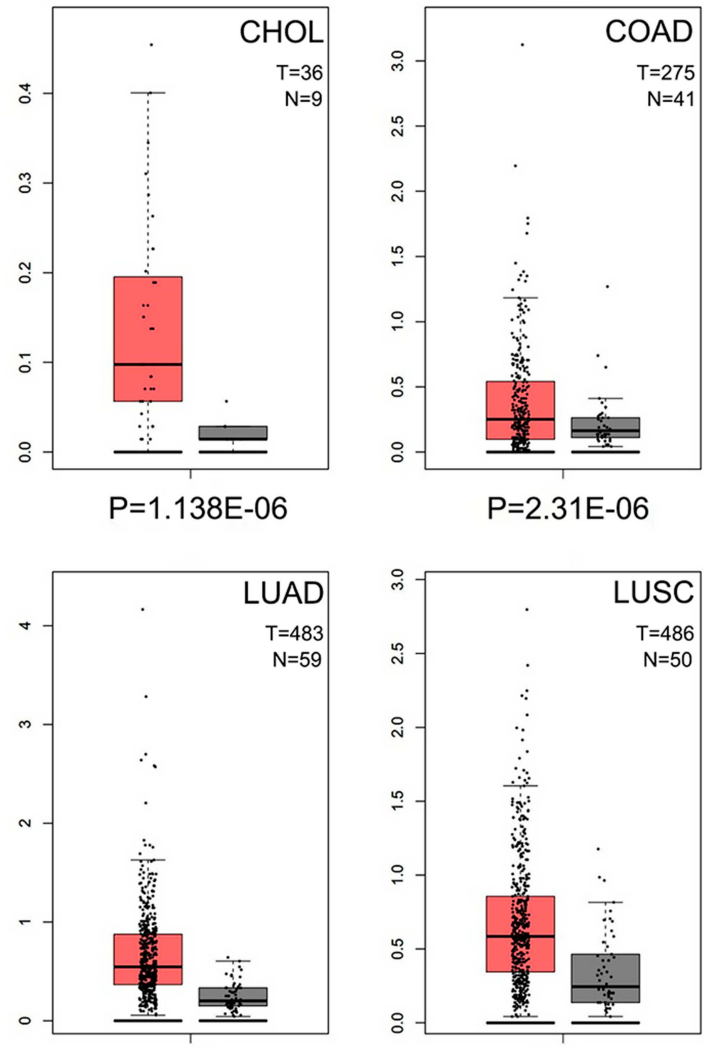

$\mathrm{P}=1.625 \mathrm{E}-12$

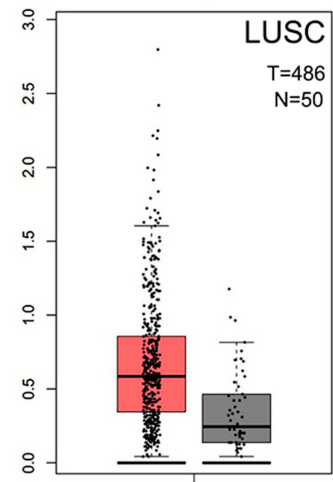

$\mathrm{P}=5.44 \mathrm{E}-15$
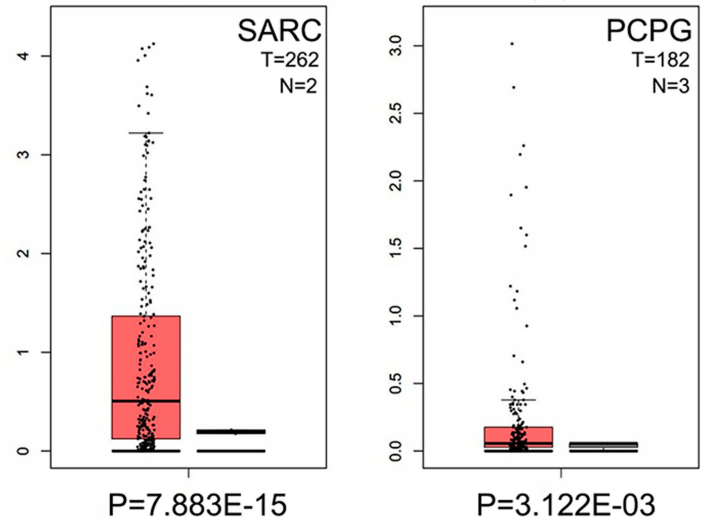

Figure 8 COL24AI expression analysis in GEPIA. (A) The expression of COL24AI in 10 tumor tissues were obviously higher than that in normal tissues. (B) The OS curve of all tumor samples in TCGA validated that high expression of COL24AI indicated poor prognosis.

suggested that COL24A1 may play a similar role in different tumors. However, the underlying mechanisms must be confirmed with more in vivo and in vitro studies.

As confirmed in Tables 3 and 4, univariate and multivariate analysis indicated COL24A1 expression as an independent prognostic factor. In addition, we grouped the patients from the TCGA and Oncomine databases according to the expression of COL24A1. The results indicated that high COL24A1 expression was exactly correlated with a shorter OS of patients with $\mathrm{HCC}$, and the same trend was also found in our 40 follow-ups of clinical samples. However, as shown in Figure 5B, when we analyzed the disease-free survival of two groups of cases from the TCGA database, the $\mathrm{P}$ value was $>0.05$. However, as illustrated in the whole curve, the disease-free survival of the high COL24A1 expression group was still lower than that of the low COL24A1 expression group. Although the difference was not highly significant, this 

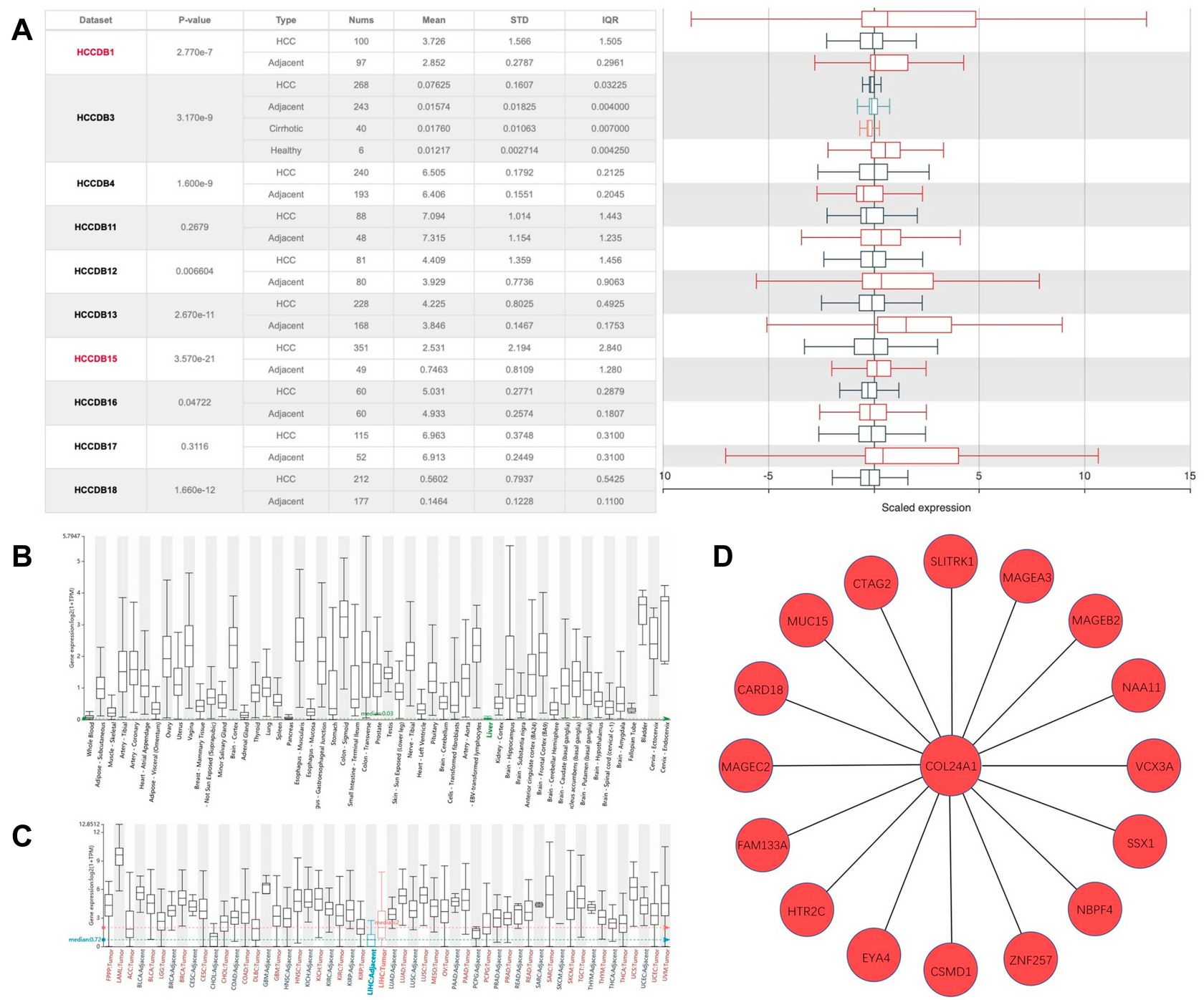

Figure 9 COL24AI expression analysis in HCCDB. (A) COL24AI expression in 8 of 10 clinical cohorts has significant differences between HCC and NH tissues. (B) The expression of col24al in normal liver tissues was very low. (C) The expression of COL24AI varied in different tissues and tumors, and the difference was significant in hepatocellular carcinoma. (D) HCC meta co-expression network about COL24AI in HCCDB.

finding may be useful for reference. Finally, the ROC (Figure 6A) analysis demonstrated COL24A1 had a remarkable diagnostic value for $\mathrm{HCC}(\mathrm{AUC}=0.75$; $\mathrm{P}<0.001)$.

To date, no specific studies have reported the correlation of COL24A1 expression and other indicators. Because cooperative detection of multiple indicators can increase the diagnostic rate of HCC, we also performed GO analysis and protein interaction network analysis. We found that COL25A1, COL15A1, COL2A1, COL6A6, COL9A1, and $\mathrm{DCN}$, as well as some other related genes and proteins, such as COL5A1, COL25A1, COL2A1, and COL9A1, in the PPI model were significantly associated with the OS.
Their co-expression is clearly of great significance for the prognosis of HCC.

In our study, we considered COL24A1 as an effective replacement or supplement to the currently used prognostic parameters. Simultaneously, we detected the alterations in COL24A1 in HCC to identify potential mechanisms in HCC. The results revealed that $2.1 \%$ of COL24A1 includes alterations such as missense mutations, amplifications, truncating mutations, and extensive deletions. From GO analysis, PPI network analysis, and mutation type analysis, we inferred that in tumors with late clinical stage, poor pathological classification as well as poor biological behavior, COL24A1 may interact 
with other proteins in its family, thereby transforming the expression of collagen proteins and proteoglycans such as DCN. ${ }^{36}$ Changes in these protein components may affect different biological activities of tumors, such as occurrence, development, invasion, and metastasis, and may ultimately lead to different prognosis. Liver cirrhosis is one of the most important steps in the development of HCC. The variety in the expression of collagen family may be associated with the long-term cirrhosis in the occurrence and development of HCC. ${ }^{37}$ Nevertheless, although the above analysis provides a novel picture for future investigation, further studies are needed to validate the functions of COL24A1 in HCC.

\section{Conclusions}

In conclusion, COL24A1 was found to be overexpressed in $\mathrm{HCC}$ and to have remarkable diagnostic and prognostic value for HCC. It is expected to become a new diagnostic and prognostic indicator of $\mathrm{HCC}$ in the future.

\section{Ethics and Consent Statement}

The present study was approved by the Ethics Committee of The Shengjing Hospital of China Medical University (Liaoning, China), because the samples we used were medical waste, the informed consent was not required, we would keep patient data strictly confidential. In line with the declaration of Helsinki. Ethical code: 2019PS542K.

\section{Acknowledgments}

Funding: This study was supported by Department of Science \&Technology of Liaoning province project (2017225032), Science and Technology Bureau of Shenyang City project (17-230-9-16), and 345 Talent Project. The authors also would like to thank The Cancer Genome Atlas (https://cancergenome.nih.gov/), International Cancer Genome Consortium (https://icgc. org) and Oncomine (https://www.oncomine.org).

\section{Author Contributions}

(I) Conception and design: Long Yan

(II) Administrative support: Chaoliu Dai

(III) Provision of study materials or patients: Chaoliu Dai and Feng $\mathrm{Xu}$

(IV) Collection and assembly of data: Long Yan

(V) Data analysis and interpretation: Long Yan

(VI) Manuscript writing: All authors
(VII) Final approval of manuscript: All authors

All authors contributed to data analysis, drafting or revising the article, gave final approval of the version to be published, and agree to be accountable for all aspects of the work.

\section{Disclosure}

The authors report no conflicts of interest in this work.

\section{References}

1. Kim E, Lisby A, Ma C, et al. Promotion of growth factor signaling as a critical function of beta-catenin during HCC progression. Nat Commun. 2019;10(1):1909. doi:10.1038/s41467-019-09780-z

2. De Toni EN, Schlesinger-Raab A, Fuchs M, et al. Age independent survival benefit for patients with hepatocellular carcinoma (HCC) without metastases at diagnosis: a population-based study. Gut. 2019.

3. DiStefano JK, Davis B. Diagnostic and prognostic potential of AKR1B10 in human hepatocellular carcinoma. Cancers. 2019;11 (4):486. doi:10.3390/cancers11040486

4. Li YM, Liu ZY, Wang JC, et al. Receptor-interacting protein kinase 3 deficiency recruits myeloid-derived suppressor cells to hepatocellular carcinoma through the chemokine (C-X-C Motif) Ligand 1-Chemokine (C-X-C Motif) receptor 2 axis. Hepatology. 2019; 70:1564-1581.

5. Matsuo N, Tanaka S, Yoshioka H, Koch M, Gordon MK, Ramirez F. Collagen XXIV (Col24a1) gene expression is a specific marker of osteoblast differentiation and bone formation. Connect Tissue Res. 2008;49(2):68-75. doi:10.1080/03008200801913502

6. Myllyharju J, Kivirikko KI. Collagens, modifying enzymes and their mutations in humans, flies and worms. Trends Genet. 2004;20 (1):33-43. doi:10.1016/j.tig.2003.11.004

7. Misawa K, Kanazawa T, Imai A, et al. Prognostic value of type XXII and XXIV collagen mRNA expression in head and neck cancer patients. Mol Clin Oncol. 2014;2(2):285-291. doi:10.3892/ mco.2013.233

8. Yin Y, Zhao Y, Li AQ, Si JM. Collagen: a possible prediction mark for gastric cancer. Med Hypotheses. 2009;72(2):163-165. doi:10.1016/j.mehy.2008.09.010

9. Maehara J, Masugi Y, Abe T, et al. Quantification of intratumoral collagen and elastin fibers within hepatocellular carcinoma tissues finds correlations with clinico-patho-radiological features. Hepatol Res. 2019.

10. Liu Y, Zhang J, Chen Y, et al. The correlation and role analysis of COL4A1 and COL4A2 in hepatocarcinogenesis. Aging. 2020;12 (1):204-223. doi:10.18632/aging.v12i1

11. Kimura K, Nakayama M, Naito I, et al. Human collagen XV is a prominent histopathological component of sinusoidal capillarization in hepatocellular carcinogenesis. Int J Clin Oncol. 2016;21 (2):302-309. doi:10.1007/s10147-015-0888-2

12. Zhang R, Ma M, Lin XH, et al. Extracellular matrix collagen I promotes the tumor progression of residual hepatocellular carcinoma after heat treatment. BMC Cancer. 2018;18(1):901. doi:10.1186/ s12885-018-4820-9

13. Ma HP, Chang HL, Bamodu OA, et al. Collagen 1A1 (COL1A1) is a reliable biomarker and putative therapeutic target for hepatocellular carcinogenesis and metastasis. Cancers. 2019;11(6):786. doi:10.3390/ cancers 11060786

14. Matsuo N, Tanaka S, Gordon MK, Koch M, Yoshioka H, Ramirez F. CREB-AP1 protein complexes regulate transcription of the collagen XXIV gene (Col24a1) in osteoblasts. J Biol Chem. 2006;281 (9):5445-5452. doi:10.1074/jbc.M509923200 
15. Koch M, Laub F, Zhou P, et al. Collagen XXIV, a vertebrate fibrillar collagen with structural features of invertebrate collagens: selective expression in developing cornea and bone. J Biol Chem. 2003;278 (44):43236-43244. doi:10.1074/jbc.M302112200

16. Sengupta S, Parikh ND. Biomarker development for hepatocellular carcinoma early detection: current and future perspectives. Hepatic Oncol. 2017;4(4):111-122. doi:10.2217/hep-2017-0019

17. Khien VV, Mao HV, Chinh TT, et al. Clinical evaluation of lentil lectin-reactive alpha-fetoprotein-L3 in histology-proven hepatocellular carcinoma. Int J Biol Markers. 2001;16(2):105-111. doi:10.1177/ 172460080101600204

18. Qiao SS, Cui ZQ, Gong L, et al. Simultaneous measurements of serum AFP, GPC-3 and HCCR for diagnosing hepatocellular carcinoma. Hepato-Gastroenterol. 2011;58(110-111):1718-1724. doi:10.5754/hge11124

19. Zhu WW, Guo JJ, Guo L, et al. Evaluation of midkine as a diagnostic serum biomarker in hepatocellular carcinoma. Clin Cancer Res. 2013;19(14):3944-3954. doi:10.1158/1078-0432.CCR-12-3363

20. Zhou J, Yu L, Gao X, et al. Plasma microRNA panel to diagnose hepatitis B virus-related hepatocellular carcinoma. J Clin Oncol. 2011;29(36):4781-4788. doi:10.1200/JCO.2011.38.2697

21. Kamarajah SK, Frankel TL, Sonnenday C, Cho CS, Nathan H. Critical evaluation of the American Joint Commission on Cancer (AJCC) 8th edition staging system for patients with hepatocellular carcinoma (HCC): a Surveillance, Epidemiology, End Results (SEER) analysis. J Surg Oncol. 2018;117(4):644-650. doi:10.1002/ jso. 24908

22. Kim Y, Moris DP, Zhang XF, et al. Evaluation of the 8th edition American Joint Commission on Cancer (AJCC) staging system for patients with intrahepatic cholangiocarcinoma: a surveillance, epidemiology, and end results (SEER) analysis. J Surg Oncol. 2017;116 (6):643-650. doi:10.1002/jso.24720

23. Robinson MD, McCarthy DJ, Smyth GK. edgeR: a Bioconductor package for differential expression analysis of digital gene expression data. Bioinformatics. 2010;26(1):139-140. doi:10.1093/bioinformatics/btp616

24. Anders S, Huber W. Differential expression analysis for sequence count data. Genome Biol. 2010;11(10):R106. doi:10.1186/gb-201011-10-r106

25. McCarthy DJ, Chen Y, Smyth GK. Differential expression analysis of multifactor RNA-Seq experiments with respect to biological variation. Nucleic Acids Res. 2012;40(10):4288-4297. doi:10.1093/ nar/gks042
26. Ritchie ME, Phipson B, Wu D, et al. limma powers differential expression analyses for RNA-sequencing and microarray studies. Nucleic Acids Res. 2015;43(7):e47. doi:10.1093/nar/gkv007

27. Ito K, Murphy D. Application of ggplot2 to pharmacometric graphics. CPT Pharmacometrics Syst Pharmacol. 2013;2:e79.

28. Raudvere U, Kolberg L, Kuzmin I, et al. g:Profiler: a web server for functional enrichment analysis and conversions of gene lists (2019 update). Nucleic Acids Res. 2019;47(W1):W191-w8. doi:10.1093/ nar/gkz369

29. Szklarczyk D, Gable AL, Lyon D, et al. STRING v11: protein-protein association networks with increased coverage, supporting functional discovery in genome-wide experimental datasets. Nucleic Acids Res. 2019;47(D1):D607-D13. doi:10.1093/nar/gky1131

30. Lian Q, Wang S, Zhang G, et al. HCCDB: a database of hepatocellular carcinoma expression atlas. Genom Proteom Bioinf. 2018;16 (4):269-275. doi:10.1016/j.gpb.2018.07.003

31. Gao J, Aksoy BA, Dogrusoz U, et al. Integrative analysis of complex cancer genomics and clinical profiles using the cBioPortal. Sci Signal. 2013;6(269):pl1. doi:10.1126/scisignal.2004088

32. Cerami E, Gao J, Dogrusoz U, et al. The cBio cancer genomics portal: an open platform for exploring multidimensional cancer genomics data. Cancer Discov. 2012;2(5):401-404. doi:10.1158/21598290.CD-12-0095

33. Zhou K, Fountzilas C. Outcomes and quality of life of systemic therapy in advanced hepatocellular carcinoma. Cancers. 2019;11 (6):861. doi:10.3390/cancers 11060861

34. Li J, Wang J, Lei L, Yuan G, He S. The diagnostic performance of gadoxetic acid disodium-enhanced magnetic resonance imaging and contrast-enhanced multi-detector computed tomography in detecting hepatocellular carcinoma: a meta-analysis of eight prospective studies. Eur Radiol. 2019;29(12):6519-6528. doi:10.1007/s00330019-06294-6

35. Toader E, Bancu A, Mitrica DE, Constantinescu G, Stefanescu G, Balan GG. Interrelations between elevated alpha-fetoprotein levels and tumor morphology of patients with hepatocellular carcinoma. Rom J Morphol Embryol. 2019;60(1):181-187.

36. Li G, Li M, Liang X, et al. Identifying DCN and HSPD1 as potential biomarkers in colon cancer using 2D-LC-MS/MS combined with iTRAQ technology. J Cancer. 2017;8(3):479-489. doi:10.7150/ jca. 17192

37. Mittal S, El-Serag HB. Epidemiology of hepatocellular carcinoma: consider the population. J Clin Gastroenterol. 2013;47(Suppl):S2-6. doi:10.1097/MCG.0b013e3182872f29
OncoTargets and Therapy

\section{Publish your work in this journal}

OncoTargets and Therapy is an international, peer-reviewed, open access journal focusing on the pathological basis of all cancers, potential targets for therapy and treatment protocols employed to improve the management of cancer patients. The journal also focuses on the impact of management programs and new therapeutic

Submit your manuscript here: https://www.dovepress.com/oncotargets-and-therapy-journal agents and protocols on patient perspectives such as quality of life, adherence and satisfaction. The manuscript management system is completely online and includes a very quick and fair peer-review system, which is all easy to use. Visit http://www.dovepress.com/ testimonials.php to read real quotes from published authors. 